FERMINO MH; GONÇALVES RS; SILVEIRA JRP; BATTISTIN A; TREVISAN M; BUSNELLO AC. 2014. Fibra de palmeira como substrato para hortaliças. Horticultura Brasileira 32: 404-408. DOI - http://dx.doi.org/10.1590/S0102-053620140000400006

\title{
Fibra de palmeira como substrato para hortaliças
}

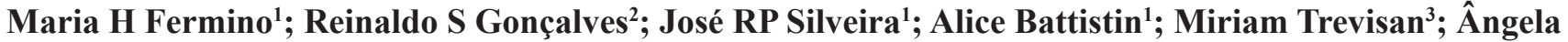 \\ C Busnello ${ }^{3}$ \\ ${ }^{1}$ Fundação Est. Pesq. Agropec. do Rio Grande do Sul, R. Gonçalves Dias 570, Menino Deus, 90130-060 Porto Alegre-RS; ${ }^{2}$ UFRGS, \\ C. Postal 15049; 91501-970 Porto Alegre-RS; maria-fermino@fepagro.rs.gov.br; reinaldo@iq.ufrgs.br; alice-battistin@fepagro.rs.gov. \\ br; jose-silveira@fepagro.rs.gov.br; miriamagronomia@gmail.com; acbusnello@yahoo.com.br; ${ }^{3}$ UFRGS-Instituto de quimica, C.Postal \\ 15049, 91501-970 Porto Alegre-RS
}

\section{RESUMO}

Este trabalho teve por objetivo estudar a possibilidade de aproveitamento dos resíduos provenientes da indústria de conserva de palmito como substrato para hortaliças. Assim, a fibra da palmeira real australiana (Archontophoenix alexandrae) foi utilizada na forma in natura (FN), coletada imediatamente após a extração do palmito e, após sofrer processo de hidrólise ácida $(\mathrm{FH})$, nas proporções 1:1:1 (v:v:v) de folha, "cartucho" (bainhas) e estipe. Os materiais puros $(100 \% \mathrm{FN}$ e $100 \% \mathrm{FH})$ e suas misturas com turfa marrom in natura, em proporção de volume $(80: 20,60: 40,40: 60,20: 80$ e 0:100\%), foram submetidos às analises físicas (densidade úmida e seca, porosidade total, espaço de aeração e disponibilidade de água) e químicas (valor de $\mathrm{pH}$ e condutividade elétrica), em delineamento inteiramente casualizado, com três repetições. Todos os resultados foram submetidos à análise de variância e, quando estatisticamente significativos, à análise de regressão. Tanto FN quanto FH puros, apresentaram baixa densidade, altos valores de porosidade total e espaço de aeração e baixa retenção de água. Em todas as misturas houve redução da densidade. A adição de FN à turfa manteve alta a porosidade total, aumentou o espaço de aeração e reduziu a disponibilidade de água. FH quando adicionado à turfa reduziu a porosidade e a disponibilidade de água e aumentou o espaço de aeração. FN e FH também elevaram o valor de $\mathrm{pH}$ da turfa. No entanto, a adição de FN elevou a condutividade elétrica, enquanto que, a adição de $\mathrm{FH}$ a diminuiu significativamente. Considerando-se a disponibilidade dos resíduos da palmeira e as excelentes características do material após a hidrólise ácida, este poderá se constituir em um material básico para formulação de substratos.

Palavras-chave: Archontophoenix alexandrae, palmeira real australiana; turfa, hidrólise ácida, características físicas e químicas.

\section{ABSTRACT \\ Palm fiber as substrate for vegetables}

This research aimed to study the possibility of recovery of waste from the canning industry of palm heart as substrates for vegetables. The fiber of Australian king palm (Archontophoenix alexandrae) was used in natura $(\mathrm{FN})$, collected immediately after the heart extraction, and after the acid hydrolysis process (FH), in the proportions 1:1:1 (v:v:v) leaf, "cartridge" (sheaths) and stipes. Materials in pure form $(100 \% \mathrm{FN}$ and $100 \% \mathrm{FH})$ and their mixtures with in natura brown peat, in volume proportions (80:20, 60:40, 40:60, 20:80 and $0: 100 \%$ ), were submitted to physical (moist and dry bulk density, total porosity, air space and available water), and chemical analyses ( $\mathrm{pH}$ and electric conductivity), in completely randomized design with three repetlications. All results were submitted to variance analysis and, when statistically significant, to regression analysis. Both pure $\mathrm{FN}$ and FH presented low dry density, high values for total porosity and air space and low water retention. All mixtures with peat showed density reduction. The addition of FN to peat increased total porosity, air space, and reduced available water. FH added to peat reduced the total porosity and available water, and increased the air space. FN and $\mathrm{FH}$ also increased the peat $\mathrm{pH}$ value. However, the addition of FN increased the electric conductivity, while the addition of FH decreased it significantly. Considering the availability of palm residues and its excellent characteristics after the acid hydrolysis, this may constitute a basic material for the formulation of substrates.

Keywords: Archontophoenix alexandrae, Australian king palm, peat, acid hydrolysis, physical characteristics, chemical characteristics.

(Recebido para publicação em 6 de setembro de 2013; aceito em 1 de agosto de 2014) (Received on September 6, 2013; accepted on August 1, 2014)

\begin{abstract}
$\mathrm{A}_{\mathrm{e}}^{\mathrm{N}}$ Mata Atlântica abriga nove gêneros e trinta e nove espécies de palmeiras, sendo a Juçara (Euterpe edulis), de onde se obtém o palmito, uma espécie nativa que cresce no estrato inferior da floresta. A exploração do palmito sempre se deu de forma extrativista, buscando a matéria-prima nas reservas naturais existentes, sem haver preocupação com
\end{abstract}

o repovoamento das áreas exploradas. Consequentemente, a palmeira nativa Euterpe edulis, tornou-se escassa e com grande possibilidade de ser extinta. Com a redução desta palmeira, outras espécies passaram a ser estudadas, na busca de plantas mais precoces e com um produto da qualidade do palmito obtido com a palmeira nativa. Dentre elas, tem merecido destaque a palmeira real australiana (Archontophoenix alexandrae).

A colheita do palmito é feita após um tempo médio de cultivo que varia de 4 anos, para a palmeira real australiana, a até 8 anos, para as nativas como a juçara. De cada planta colhida extrai-se cerca de 400 g de palmito comercial, e gera-se aproximadamente $13 \mathrm{~kg}$ de resíduos 
compostos por estipes e folhas que ficam no campo e por bainhas ("cartucho" que envolve o palmito) que ficam no chão da fábrica de conserva, sem aproveitamento econômico.

As plantas olerícolas, florestais, frutíferas, ornamentais, medicinais, aromáticas e mesmo culturas industriais como fumo, necessitam em pelo menos algumas fases do desenvolvimento, do cultivo em recipientes (bandejas, sacos, vasos, tubetes, entre outros). $\mathrm{O}$ meio onde se desenvolvem as raízes, em substituição ao solo in situ e que, portanto, serve de suporte à planta, denomina-se substrato (Kämpf, 2000). Existem referências em literatura do uso de diversos materiais componentes como estercos, fibras naturais, lixo urbano, solo, poliuretanos, aguapé, bagaço de cana, "confete" de papel (resíduo da fabricação de formulário contínuo), maravalha, serragem, cascas de árvores, casca de arroz e até mesmo pneu picado (carcaça lixada de pneu para recapeamento), que foram analisados do ponto de vista do uso como substrato para plantas. Um dos exemplos mais recentes e de grande abrangência no país é a fibra da casca do coco (endocarpo). Até então constituía um "poluente" do ambiente sendo utilizada em baixa escala pela indústria para confecção de bancos de automóveis. A partir da determinação do potencial do material como substrato, as grandes empresas, que até então cultivavam o coqueiro para obtenção do coco e da água, passaram a implantar áreas de cultivo com o objetivo de obter matéria-prima para as fábricas de substrato. Este substrato tem despertado interesse como substituto de materiais considerados excelentes como a turfa e a fibra de xaxim.

O presente estudo teve por objetivo avaliar as características físicas e químicas do resíduo da extração do palmito da palmeira real australiana com vistas a sua utilização como substrato para plantas.

\section{MATERIAL E MÉTODOS}

O trabalho foi executado no Laboratorio de Analises de Substrato para Plantas (LASPP), da Fundação Estadual de Pesquisa Agropecuária do Rio
Grande do Sul, de 2004 a 2006. A fibra da palmeira real australiana constitui resíduo da indústria de palmito em conserva e foi fornecida pela empresa Natusol, localizada no município de Vale do Sol-RS.

A fibra foi utilizada nas proporções 1:1:1 (v:v:v) de folha, "cartucho" (bainhas) e estipe, das seguintes formas: 1) fibra in natura (FN) - as fibras coletadas imediatamente após a extração do palmito, foram trituradas até atingirem a espessura aproximada de um $\mathrm{cm} ; 2$ ) fibra hidrolisada (FH) - as fibras após trituradas sofreram processo de hidrólise ácida a 1\% (v/v) de ácido sulfúrico por 30 minutos de autoclavagem na temperatura de $120^{\circ} \mathrm{C}$ e pressão de 1,5 atm, neutralização e sucessivas lavagens. A hidrólise teve como objetivo a extração de açúcar com fins de produção de álcool carburante (Battistin et al., 2006).

Misturas foram compostas com turfa marrom in natura $(\mathrm{T})$, proveniente da empresa Florestal SA de Araranguá-SC, com fibra de palmeira, in natura (FN) e hidrolisada $(\mathrm{FH})$

Os materiais puros e suas misturas com turfa foram submetidos às analises físicas (densidade úmida e seca, porosidade total, espaço de aeração e disponibilidade de água) e químicas (valor de $\mathrm{pH}$ e condutividade elétrica), como descritas abaixo.

A determinação da densidade seca foi realizada através do método da autocompactação adaptado de Hoffmann (1970) e recomendado pela Federação dos Institutos para Pesquisas e Análises Agrícolas, VDLUFA (Alemanha) (Röber \& Schaller, 1985) e ALVA (Áustria) (Baumgarten, 2002), para análise de substratos.

O método prevê o preenchimento de uma proveta plástica, transparente e graduada, de $500 \mathrm{~mL}$, com aproximadamente $300 \mathrm{~mL}$ de substrato na umidade atual. Após, esta proveta é deixada cair solta, sob a ação do seu próprio peso, de uma altura de $10 \mathrm{~cm}$ por 10 (dez) vezes consecutivas. Com auxílio de uma espátula nivela-se a superfície levemente, e lê-se o volume obtido ( $\mathrm{mL}$ ). Em seguida pesa-se o material úmido e leva-se à estufa para secagem a $65^{\circ} \mathrm{C}$ até peso constante (ou 48 horas).

A determinação da porosidade total, espaço de aeração, água facilmente disponível e água tamponante foi realizada através de curvas de retenção de água nas tensões de $0,10,50$, e $100 \mathrm{~cm}$ de altura de coluna de água, correspondendo às pressões de $0 ; 0,1 ; 0,5$ e $1,0 \mathrm{kPa}$, conforme os princípios de De Boodt \& Verdonck (1972).

Os valores foram obtidos pelo método do funil proposto por Vomicil (apud Gauland, 1997) e adaptados em Fermino (2003). A construção das curvas de retenção de água foi efetuada com os valores de umidade volumétrica obtidos através dos percentuais de água retida para cada tensão.

Para a determinação do valor de $\mathrm{pH}$ e da condutividade elétrica (CE) utilizou-se o método recomendado pela CEN (European Committee for Standardization) (European Standard EN 13037), segundo Miner (1994) com diluição 1:5 (v:v) de substrato:água deionizada. Após, determinada a densidade, foi pesada uma massa da amostra equivalente a uma alíquota de $60 \mathrm{~mL}$ e transferida para um frasco de $500 \mathrm{~mL}$. Adicionou-se $300 \mathrm{~mL}$ de água, tampou-se e agitou-se à rotação de 40 rpm por 1 hora.

O delineamento experimental foi inteiramente casualizado, em esquema fatorial com 12 tratamentos: duas misturas, FN:T e FH:T, e seis proporções de mistura (100:0; 80:20; 60:40; 40:60; 20:80; 0:100\%), com três repetições. As variáveis físicas e químicas foram submetidas à análise de variância e, quando significativas, à análise de regressão. Utilizou-se o Programa Sigma Stat 3.2.

\section{RESULTADOS E DISCUSSÃO}

Das variáveis físicas e químicas estudadas, à exceção da variável Água tamponante (AT) da fibra hidrolisada (FH), todas as demais apresentaram valores estatisticamente significativos: Densidade seca (DS), Porosidade total (PT), Espaço de aeração (EA) e Água facilmente disponível (AFD).

As variáveis Densidade seca (DS), Porosidade total (PT), Espaço de aeração (EA) e Água facilmente disponível (AFD) da fibra in natura (FN) foram analisadas com base nos valores ori- 


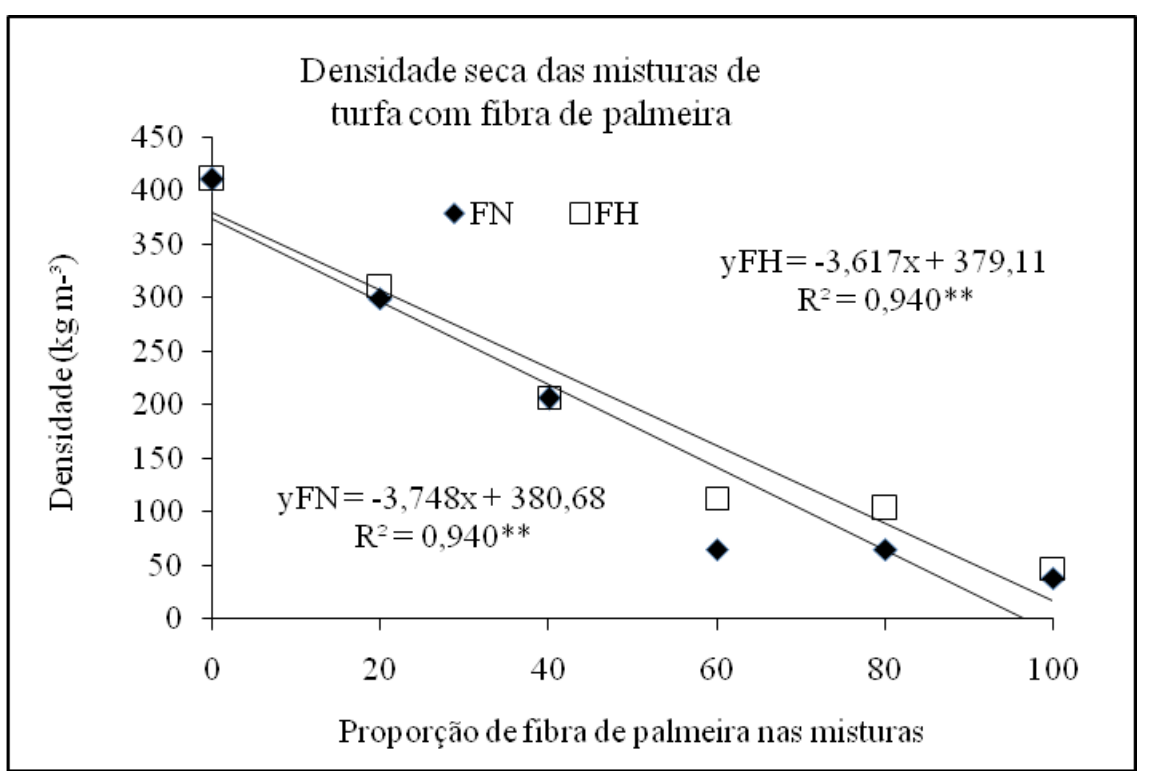

Figura1. Análise de regressão da densidade seca $\left(\mathrm{kg} / \mathrm{m}^{3}\right)$ das misturas de turfa marrom in natura com fibra in natura de palmeira (FN) e com fibra hidrolisada de palmeira (FH) (\% do volume) (média de três amostras) (todos parâmetros das equações foram significativos a $1 \%$ de probabilidade pelo teste $\mathrm{t}$ ) [regression analysis of dry density $\left(\mathrm{kg} / \mathrm{m}^{3}\right)$ with mixtures of in natura brown peat with in natura palm tree fiber (FN) and with hydrolysed palm tree fiber $(\mathrm{FH})(\%$ of the volume) (average of three samples) (all parameters of the equations were significant at $1 \%$ probability by the $t$ test)]. Porto Alegre, FEPAGRO, 2007.

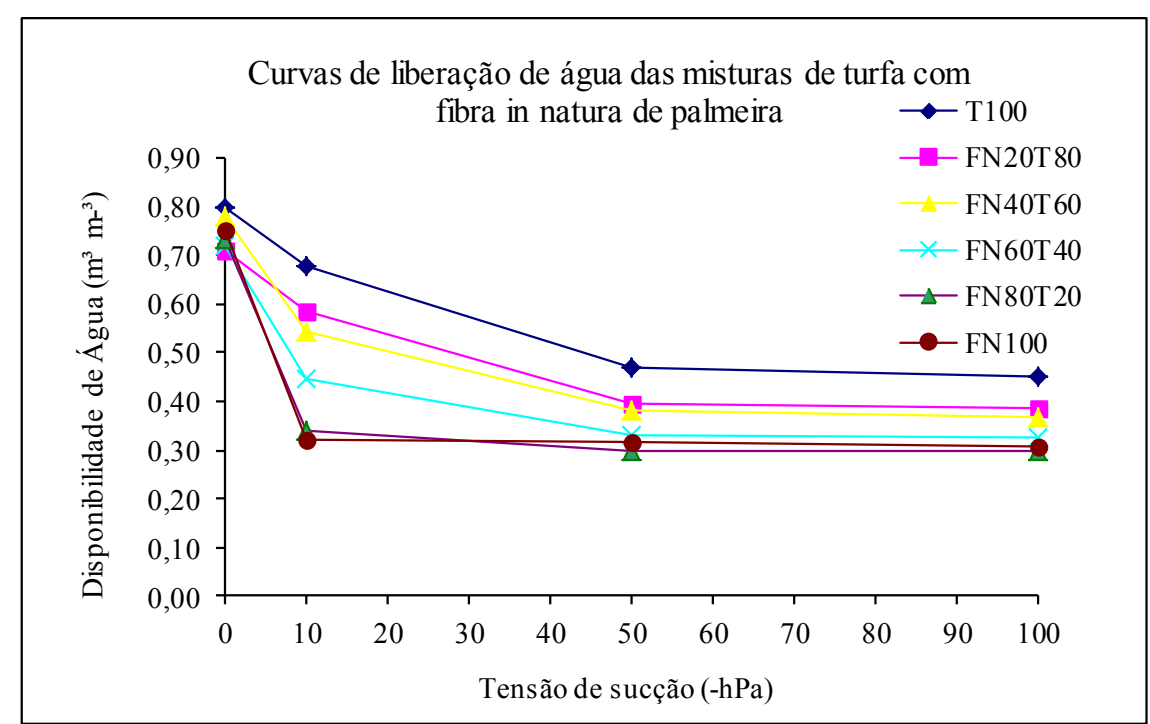

Figura 2. Curvas de liberação de água $\left(\mathrm{m}^{3} / \mathrm{m}^{3}\right)$ das misturas de turfa marrom in natura $(\mathrm{T})$ com fibra in natura de palmeira (FN) (\% do volume) (média de três amostras) [curves of water release $\left(\mathrm{m}^{3} / \mathrm{m}^{3}\right)$ in mixtures of in natura brown peat $(\mathrm{T})$ with in natura palm tree fiber (FN) (\% of the volume) (average of three samples)]. Porto Alegre, FEPAGRO, 2007.

ginais enquanto os valores da Água tamponante (AT) sofreram transformação $(\sqrt{y})$.

As variáveis Densidade seca (DS), Porosidade total (PT) e Água facilmente disponível (AFD) da fibra hidrolisada (FH) foram analisadas com base nos valores originais enquanto os valores de Espaço de aeração (EA) sofreram transformação $(\sqrt{y})$.

Das variáveis químicas estudadas, a Condutividade elétrica (CE) de FN teve seus dados transformados $(\sqrt{(\mathrm{y}+1 / 2})$.

FN apresentou baixo valor de DS $\left(38 \mathrm{~kg} / \mathrm{m}^{3}\right)$, menor do que o valor de $\mathrm{FH}$ $\left(46 \mathrm{~kg} / \mathrm{m}^{3}\right)$ e ambos menores do que o valor da turfa marrom $(\mathrm{T})\left(410 \mathrm{~kg} / \mathrm{m}^{3}\right)$ (Figura 1).

As primeiras referências a valores de densidade foram feitas por Bunt (1973), sugerindo entre 400 e $500 \mathrm{~kg} /$ $\mathrm{m}^{3}$; atualmente, entende-se que o valor da densidade deve ser adequado à exigência da planta, das condições de irrigação e, principalmente, da altura/ volume do recipiente.

Assim, quanto menor o recipiente, menor dever ser a densidade do substrato nele utilizado. Kämpf (2000) recomenda utilizar substratos com densidade de 100 a $300 \mathrm{~kg} / \mathrm{m}^{3}$ para bandejas multicelulares, de 250 a $400 \mathrm{~kg} / \mathrm{m}^{3}$ para vasos de até $15 \mathrm{~cm}$ de altura, de $300 \mathrm{a}$ $500 \mathrm{~kg} / \mathrm{m}^{3}$ para vasos de 20 a $30 \mathrm{~cm}$, e, de 500 a $800 \mathrm{~kg} / \mathrm{m}^{3}$ para vasos maiores de $30 \mathrm{~cm}$. Martínez (2002) sugere valores de densidade de substrato entre 500 e $750 \mathrm{~kg} / \mathrm{m}^{3}$ para recipientes colocados ao ar livre e, menores de $150 \mathrm{~kg} / \mathrm{m}^{3}$ para cultivos em estufa.

Poucos são os materiais com valores tão baixos de densidade, o que favorece o uso da fibra de palmeira como condicionador de substratos com alta densidade, condição mais comum dentro da atividade hortícola. Situação semelhante é encontrada quando das misturas com turfa marrom, onde houve redução linear da densidade das misturas com o incremento das fibras (Figura 1).

Tanto FN quanto FH apresentaram altos valores de PT e de EA, sendo os valores de FN menores do que FH (Figuras 2 e 3 ).

FN apresentou valor de PT $\left(0,75 \mathrm{~m}^{3} /\right.$ $\left.\mathrm{m}^{3}\right)$ ligeiramente inferior ao da $\mathrm{T}(0,80$ $\mathrm{m}^{3} / \mathrm{m}^{3}$ ). Nas misturas entre FN e T, independentemente dos percentuais, os valores de PT foram constantes e altos, porém menores do que os valores dos materiais puros (Figura 2).

$\mathrm{O}$ valor de PT de FH $\left(0,85 \mathrm{~m}^{3} /\right.$ $\left.\mathrm{m}^{3}\right)$ é superior ao de $\mathrm{T}\left(0,80 \mathrm{~m}^{3} / \mathrm{m}^{3}\right)$, e as misturas, independentemente dos percentuais, apresentaram valores de PT inferiores aos materiais puros. Esta redução foi mais significativa na mistura FH40T60 $\left(0,54 \mathrm{~m}^{3} / \mathrm{m}^{3}\right)$ (Figura 3).

Considera-se desejável que o valor de PT de um substrato situe-se em 


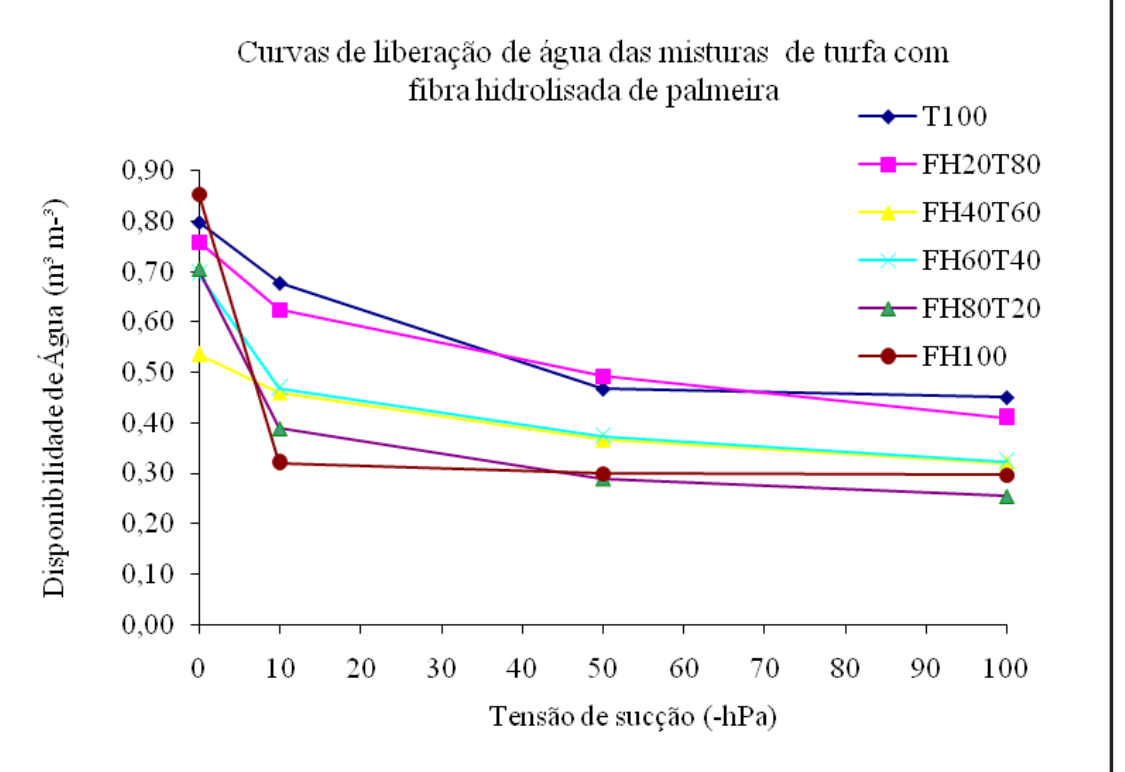

Figura 3. Curvas de liberação de água $\left(\mathrm{m}^{3} / \mathrm{m}^{3}\right)$ das misturas de turfa marrom in natura com fibra hidrolisada de palmeira (FH) (\% do volume) (média de três amostras) [curves of water release $\left(\mathrm{m}^{3} / \mathrm{m}^{3}\right)$ in mixtures of in natura brown peat with hydrolysed palm tree fiber $(\mathrm{FH})(\%$ of the volume) (average of three samples)]. Porto Alegre, FEPAGRO, 2007.

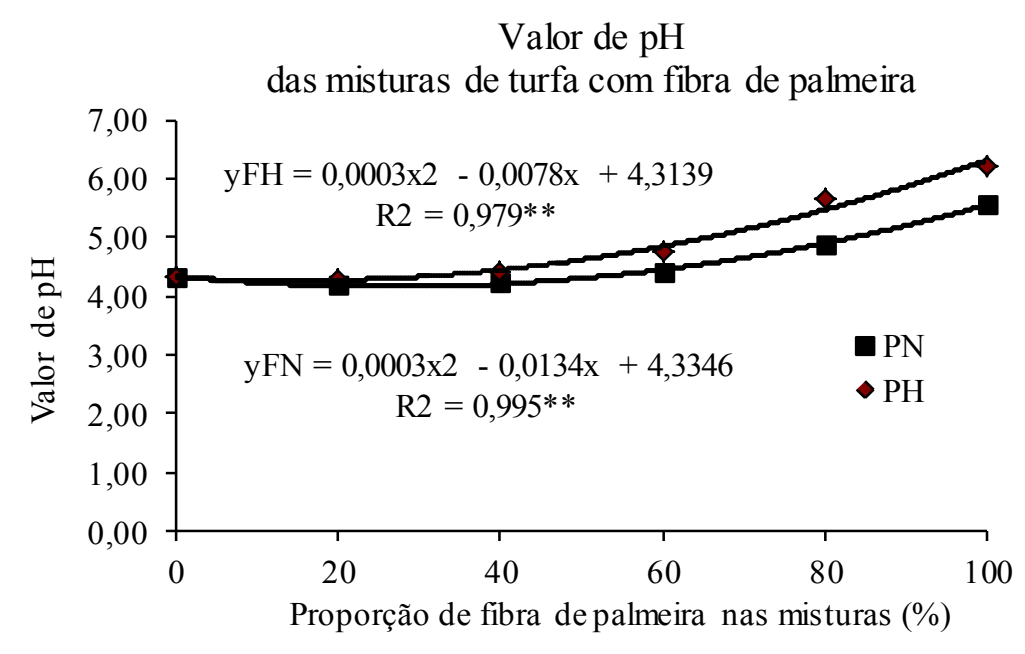

Figura 4. Análise de regressão dos valores de $\mathrm{pH}$ das misturas de turfa marrom in natura com fibra in natura de palmeira (FN), e de turfa marrom in natura com fibra hidrolisada de palmeira (FH) (\% do volume) (média de três amostras) (todos parâmetros das equações foram significativos a $1 \%$ de probabilidade pelo teste $\mathrm{t}$ ) [regression analysis of $\mathrm{pH}$ values of mixtures of brown peat in natura with in natura palm tree fiber (FN), and in natura brown peat with hydrolysed palm tree fiber (FH) ( $\%$ of the volume) (average of three samples) (all parameters of the equations were significant at $1 \%$ probability by the $t$ test)]. Porto Alegre, FEPAGRO, 2007.

torno de $85 \%$ (De Boodt \& Verdonck, 1972; Boertje, 1984; Verdonck \& Gabriels, 1988). No entanto, Handreck \& Black (1999) afirmam que o espaço poroso total é importante, mas a forma e o tamanho dos poros são ainda mais importantes. Mais importante do que o volume de poros totais é a relação entre o volume de água e de ar presente no substrato. Esta relação é determinada pelo tamanho dos poros e pela forma como estes se interligam.
$\mathrm{O}$ valor de EA de FN $\left(0,43 \mathrm{~m}^{3} / \mathrm{m}^{3}\right)$ é 3,5 vezes superior ao de $\mathrm{T}\left(0,12 \mathrm{~m}^{3} /\right.$ $\mathrm{m}^{3}$ ). Houve um acréscimo do valor de EA proporcional ao incremento de FN nas misturas (Figura 2).

O valor do EA de FH $\left(0,53 \mathrm{~m}^{3} / \mathrm{m}^{3}\right)$ é 4,4 vezes superior ao de $T\left(0,12 \mathrm{~m}^{3} / \mathrm{m}^{3}\right)$. À exceção de FH40T60, observou-se um incremento do valor de EA diretamente proporcional ao incremento de $\mathrm{FH}$ nas misturas (Figura 3).

Os valores referenciais sugeridos em literatura situam-se entre 20 e $40 \%$ do volume $\left(0,20\right.$ e $\left.0,40 \mathrm{~m}^{3} / \mathrm{m}^{3}\right)$ (DeBoodt \& Verdonck, 1972; Penningsfeld, 1983). No entanto, a escolha de um substrato, com um determinado valor de EA, deve ser feita considerando-se a espécie cultivada, a frequência e o tipo de irrigação, e as características do ambiente de cultivo, se natural ou protegido (Bunt, 1984; Armitage, 1994).

Substratos com altos valores de EA, como a fibra de palmeira, são recomendados para uso em recipientes pequenos como bandejas multicelulares, de maneira a aumentar a drenagem limitada pela pequena altura do recipiente (Puchalski \& Kämpf, 2000).

Segundo De Boodt \& Verdonck (1972) e Haynes \& Goh (1978) para a AFD (volume de água liberado entre 10 e $50 \mathrm{~cm}$ de tensão ou entre 0,1 e $0,5 \mathrm{kPa}$ ), entende-se como desejáveis valores entre 20 e $30 \%\left(0,20\right.$ e $\left.0,30 \mathrm{~m}^{3} / \mathrm{m}^{3}\right)$. Para os mesmos autores para AT (volume de água liberado entre 50 e $100 \mathrm{~cm}$ de tensão ou entre 0,5 e 1,0 kPa), estima-se que 4 a $10 \%\left(0,04\right.$ a $\left.0,1 \mathrm{~m}^{3} / \mathrm{m}^{3}\right)$ seja um valor de referência.

Considerando-se que a curva de retenção de água para substratos é análoga ao nível de umidade normalmente mantido em cultivos em recipientes, sob estufa, situando-se entre 10 e $100 \mathrm{~cm}$ de tensão (0,1 e 1,0 kPa) (Puustjarvi \& Robertson apud Fonteno et al., 1981), percebe-se que os materiais estudados não armazenam água suficiente para liberar durante o cultivo, necessitando pois escolha cuidadosa do sistema de irrigação.

$\mathrm{O}$ valor de $\mathrm{pH}$ de $\mathrm{FN}(5,6)$ é inferior ao de $\mathrm{FH}(6,2)$ e ambos superiores ao de $\mathrm{T}(4,3)$ (Figura 4). Apenas quando FN compôs a mistura em $80 \%$ houve uma elevação do valor de $\mathrm{pH}$ da mistura $\mathrm{o}$ 


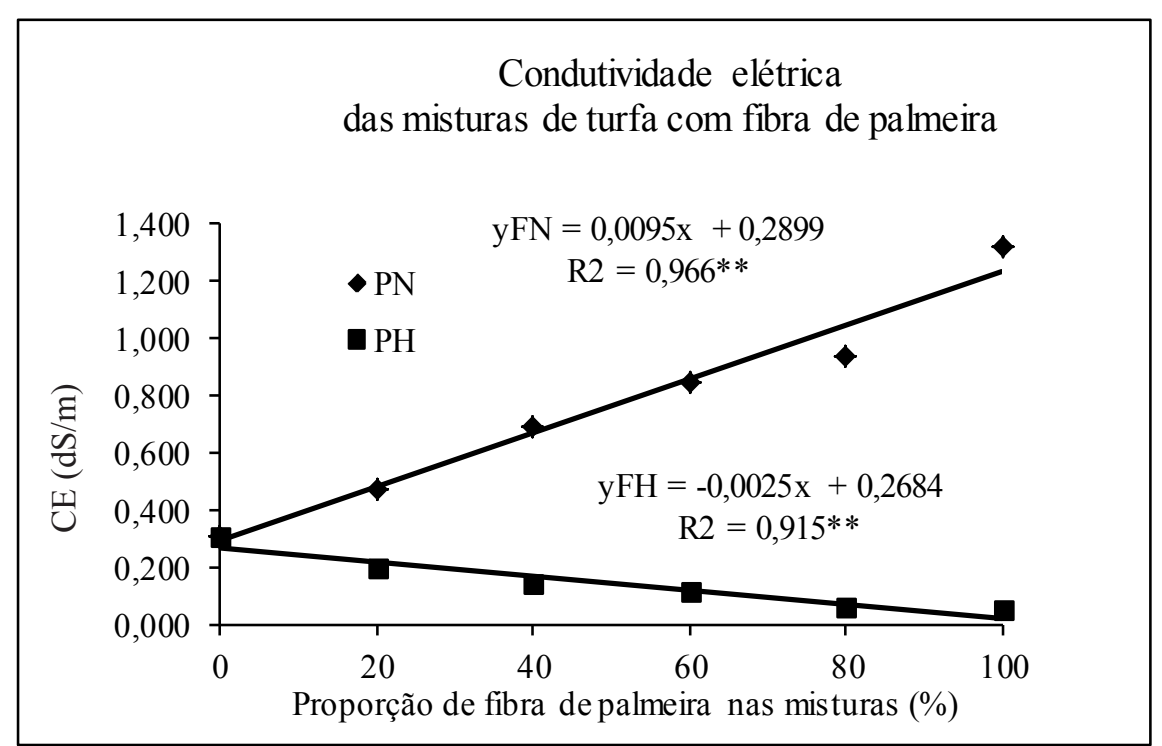

Figura 5. Análise de regressão da condutividade elétrica $(\mathrm{dS} / \mathrm{m})$ das misturas de turfa marrom in natura com fibra in natura de palmeira (FN) e de turfa marrom in natura com fibra hidrolisada de palmeira (FH) (\% do volume) (média de três amostras) (todos parâmetros das equações foram significativos a $1 \%$ de probabilidade pelo teste $\mathrm{t}$ ) [regression analysis of electrical conductivity $(\mathrm{dS} / \mathrm{m})$ mixtures of in natura brown peat with in natura palm tree fiber (FN), and in natura brown peat with hydrolysed palm tree fiber $(\mathrm{FH})(\%$ of the volume) (average of three samples) (all parameters of the equations were significant at $1 \%$ probability by the $t$ test)]. Porto Alegre, FEPAGRO, 2007.

que evidencia o alto poder tampão de T. Paralelamente, quando FH foi adicionada em 60 e $80 \%$ pode-se observar um aumento no valor de $\mathrm{pH}$ da mistura.

O valor da CE de FN $(1,318 \mathrm{dS} / \mathrm{m})$ é superior ao de $\mathrm{T}(0,307 \mathrm{dS} / \mathrm{m})$ e ao de $\mathrm{FH}$ $(0,50 \mathrm{dS} / \mathrm{m})$ (Figura 5). O incremento de FN elevou linear e significativamente o valor da CE das misturas, enquanto que, o incremento de FH diminuiu.

$\mathrm{O}$ estudo permite concluir que as fibras da palmeira real australiana, in natura ou hidrolisadas, possuem características físicas adequadas para uso como substrato. No entanto, quando se observa o resultado das características químicas estudadas conclui-se que, apesar dos valores de $\mathrm{pH}$ estarem adequados, os valores da condutividade elétrica de FN são altos para uso como substrato. Avaliações com misturas com outros materiais e em cultivo necessitam ser realizadas.

\section{AGRADECIMENTOS}

À FAPERGS e à FINEP pelo finan- ciamento do projeto de pesquisa. As Busnello agradecem a concessão de bolsa. À empresa Natusol pela cedência das fibras de palmeira utilizadas no estudo. À empresa Florestal SA pela cedência da turfa marrom utilizada no estudo.

\section{REFERÊNCIAS}

ARMITAGE AM. 1994 Ornamental bedding plants. Wallingford: CAB International. 175p. (Crop Production Science in Horticulturae, 4).

BATTISTIN A; FERMINO MH; SILVEIRA JRP; GONÇALVES RS. dez. 2007. Processo de produção por hidrólise ácida a partir da planta de palmeira de inulina e substrato para plantas. Disponível em: $<$ https://gru.inpi.gov. $\mathrm{br} / \mathrm{pPI} /$ servlet/PatenteServletController?Acti on $=$ detail $\&$ CodPedido $=726280 \&$ PesquisaPo rTitulo $=$ INULINA\&PesquisaPorResumo $=\&$ PesquisaPorDepositante $=\&$ PesquisaPorInve ntor $=\&$ PesquisaPorProcurador $=>$ Acessado em 20 jun. 2014.

BAUMGARTEN A. 2002. Methods of chemical and physical evaluation of substrates for plants. In: FURLANI AMC (ed) Caracterização, manejo e qualidade de substratos para produção de plantas. Campinas: Instituto Agronômico. 122p. (Documentos IAC, 70). BOERTJE GA. 1984. Physical laboratory analises autoras Miriam Trevisan e Ângela C of potting composts. Acta Horticulturae 150 : 47-50.

BUNT AC. 1973. Some physical and chemical characteristics of loamless pot-plant substrates and their relation to plant growth. Plant and Soil 38: 1954-1954.

BUNT AC. 1984. Physical properties of mixtures of peats and minerals of different particle size and bulk density for potting substrates. ActaHorticulturae 150: 143-153.

De BOODT M; VERDONCK O. 1972. The physical properties of the substrates in horticulture. Acta Horticulturae 26: 37-44.

FERMINO MH. 2003. Métodos para caracterização física de substratos para plantas. Porto Alegre: UFRGS. 90p. (Tese doutorado.

FONTENO WC; CASSEL DK; LARSON RA. 1981. Physical properties of three container media and their effect on poinsettia growth. Journal of the American Society for Horticultural Science 106: 736-741.

GAULAND DCSP. 1997. Relações hídricas em substratos à base de turfas sob o uso dos condicionadores casca de arroz carbonizada ou queimada. Porto Alegre: UFRGS. 107p. (Dissertação mestrado).

HANDRECK KA; BLACK ND. 1999. Growing media for ornamental plants and turf. Sydney: Unsw Press, 448p.

HAYNES RJ; GOH KM. 1978. Evaluation of potting media for commercial nursery production of container-grown plants. IV: Physical properties of a range amendment peat-based media. New Zealand Journal of Agricultural Research 21: 449-456.

HOFFMANN G. 1970. Verbindliche Methoden zur Untersuchung von TKS und Gärtnerischen Erden. Mitteilungen der VDLUFA 6: 129-153.

KÄMPF AN. 2000. Produção Comercial de Plantas Ornamentais. Guaíba: Agropecuária, 254p.

MARTÍNEZ PF. 2002. Manejo de substratos para horticultura. In: FURLANI AMC (eds). Caracterização, manejo e qualidade de substratos para produção de plantas. Campinas: Instituto Agronômico, 122p. (Documentos IAC, 70).

MINER AM. 1994. Substratos: propriedades y caracterización. Madrid: Ediciones MundiPrens. 172p.

PENNINGSFELD F. 1983. Kultur substrate für den Gartenbau, besonders in Deutschland: Ein Kritischer Überblick. Plant and Soil 75: 269-281.

PUCHALSKI LEA; KÄMPF AN. 2000. Efeito da altura do recipiente sobre a produção de mudas de Hibiscus rosa-sinensis em plugs. In: KÄMPF AN; FERMINO MH (eds). Substrato para plantas: a base da produção vegetal em recipientes. Porto Alegre: Gênesis. 312p.

RÖBER R; SCHALLER K. 1985. Pflanzenernährung im Gartenbau. Stuttgart: Ulmer. 352p.

VERDONCK O; GABRIELS R. 1988. Substrate requeriments for plants. Acta Horticulturae 221: 19-23. 